\title{
Using new evidence is an innovation. So what?
}

\section{Dominic Hurst}

Queen Mary University of London, Barts \& The London School of Medicine and Dentistry, Institute of Dentistry, UK.

When someone introduces us to a piece of research that suggests we do something differently in our clinical practice, do we realise that what we are dealing with is an innovation?

If we want to learn ourselves, or encourage fellow clinicians to learn, the skills to question clinical decisions, to search for evidence, to appraise it, to implement it and to evaluate it, do we realise that these are all innovations?

When we change the way we interact with our patients so that we blend our research knowledge with their values, does this strike us as an innovation?

Each is an innovation because an innovation is any thing or idea that is new to the individual. Such a thing or idea may have been around for many years even though it is new to the person coming across it - or deciding to adopt it. It may well be a brand new way of listening to music or of flying through air, but it may be as down to earth as using peat-free compost in the garden or printing your name beneath your signature in your patient records.

So what if the adoption of new practices and attitudes towards research - or the implementation of new research findings - are innovations for individual clinicians every day around the world?

\section{Diffusion of innovations}

Rogers, ${ }^{1}$ in his widely-quoted book on the diffusion of innovations, tells the story of a Peruvian village in which the public health service wanted to change the villagers' behaviour so that they would boil their water rather than drink the typhoidpolluted water from various local sources. The intention was to improve the villagers' health and prolong their lives. For two years a field worker tried to get the villagers to boil their water. It was a no-brainer, right?

Actually, the majority shunned the innovation and instead continued to drink the water from the irrigation ditch, the fountain and the public well.

Why didn't they adopt this simple and seemingly-obvious beneficial innovation? Here are a few reasons: they liked the taste of the ditch water; the village norms say that only ill people drink boiled water; germ theory was not understood and, in any case, there are other more obvious threats to the villagers, like hunger and poverty, without worrying about little organisms they can't see. Maybe they also didn't like outsiders telling them what to do.

\section{Forces pro and forces anti}

Forces act on all of us from inside and out either to hinder or encourage the adoption of an innovation. A century's worth of research has created a plethora of theories describing the diffusion of innovations through communities and organisations, and what might encourage or dissuade this process. What is clear on reading these is that we can underestimate the complexity of the process very easily. Do we evaluate the forces acting on an organisation or individual before introducing teaching of evidencebased practice? Do we evaluate in our clinics the forces both individual and practice-wide that will aid or hinder the adoption of new guidance on the management of disease? Or when we want to get colleagues interested in attending a course, do we think of what might encourage them to attend, and what might discourage them?

Recently Hopper et al. reported on a study in which they used qualitative methods to investigate how a group of general dental practitioners (GDPs) in the north west of England viewed research and whether it affected their clinical behaviour. ${ }^{2}$ Participants were found to acknowledge the importance of using research to inform their decision making. However, in reality whether or not they sought and incorporated evidence was influenced by such things as the culture of the practice in which they worked, their own inclination to adopt change, the perceived relevance of the existing research to their clinical practice and the financial viability of introducing a new intervention. Thus the effectiveness of the transfer of new knowledge (the innovation) was influenced as much by factors external to the innovation as it was to those internal to it (eg the type of knowledge on offer).

\section{Let's innovate, shall we?}

Research itself and the knowledge it imparts will remain bound within the pages of worthy journals unless we realise the importance of treating the search for it, the appraisal and its implementation as innovations. Thus I propose another innovation: that those of us interested in the furthering of evidence-based dental practice learn the skills to analyse in our own practices and organisations what hinders and what encourages the diffusion of evidence-based dentistry into our own, our students' and our colleagues' day-today clinical practice. This is a large field of study but we might start with some force field analysis ${ }^{3}$ and root cause analysis, ${ }^{4}$ both of which are tools to understand why something is or isn't working. All we have to do then is work out how to maximise the forces pro and minimise the forces anti the innovation. Happy innovating.

1. Rogers EM. Diffusion of innovations. 5th ed. New York, N.Y.: Simon \& Schuster; 2003.

2. Hopper L, Morris L, Tickle M. How primary care dentists perceive and are influenced by research. Commun Dent Oral Epidemiol 2011; 39: 97-104.

3. Mindtools. Force Field Analysis - Analyzing the pressures for and against change. 2011 [cited 2011 02/09/2011]; Available from: http://www.mindtools. com/pages/article/newTED 06.htm.

4. Mindtools. Root Cause Analysis - Tracing a problem to its origins. 2011 [cited 2011 02/09/2011]; Available from: http://www.mindtools.com/pages/article/ newTMC_80.htm.

Evidence-Based Dentistry (2011) 12, 66 doi:10.1038/sj.ebd.6400802 